\title{
The intrinsic properties of MAPbxSn1-xBr3 perovskite single crystals (Withdrawal Notice)
}

Chen Zou, Zonglong Zhu, Chun-Ying Huang, Lih Lin

Chen Zou, Zonglong Zhu, Chun-Ying Huang, Lih Y. Lin, "The intrinsic properties of MAPbxSn1-xBr3 perovskite single crystals (Withdrawal Notice)," Proc. SPIE 10529, Organic Photonic Materials and Devices XX, 105290H (21 February 2018); doi: 10.1117/12.2289172

SPIE. Event: SPIE OPTO, 2018, San Francisco, California, United States 


\section{The intrinsic properties of $\mathrm{MAPb}_{\mathrm{x}} \mathrm{Sn}_{1-\mathrm{x}} \mathrm{Br}_{3}$ perovskite single crystals (withdrawal notice)}

Chen Zou, Zonglong Zhu, Chun-Ying Huang, and Lih Y. Lin

University of Washington (United States)

Proc. SPIE 10529, 105290H (2018)

Online Publication Date: 21 February 2018

Withdrawn from Publication: 20 March 2018

Conference Date: 31 January-1 February 2018

Conference Location: San Francisco, California, United States

Conference Title: Organic Photonic Materials and Devices XX

Conference Chairs: Christopher Tabor, Francois Kajzar, Toshikuni Kaino, Yasuhiro Koike

Publisher's Note: This paper, originally published on 21February 2018, was withdrawn per author request. 